Revista Perspectiva Online: Humanas \& Sociais Aplicadas

Dezembro de 2017, Vol.7, no 20, p.82-89

ISSN: 2236-8876 (Online)

DOI: $10.25242 / 887672020171201$

\title{
PROGRAMA PERENE PARA A SAÚDE DO IDOSO
}

\author{
Heloisa Landim Gomes ${ }^{1 *}$, Cristiane Brasil ${ }^{2}$ \& André Luiz Marques Gomes ${ }^{3}$
}

\section{RESUMO}

GOMES, H.L., BRASIL, C., GOMES, A.L.M. Programa Perene Para a Saúde do Idoso. Perspectivas Online: Humanas \& Sociais Aplicadas, v.7, n.20, p.82-89, 2017.

O objetivo deste artigo esta pautado na potencialidade de um programa de atividades sistemáticas para os idosos. Estudo de caráter exploratório, com 83 idosos, de ambos os sexos, entre 60 e 85 anos, inseridos em um programa sistemático de atividades físicas denominado Vida Ativa, em Campos dos Goytacazes/RJ. Foi utilizada uma entrevistada elaborada pelos autores especificamente para verificar a percepção dos participantes sobre indicadores de saúde, prática de atividades, satisfação, qualidade de vida e pré-existência de doenças crônicas 6 meses após o início da participação no programa.
Os resultados mostraram alto índice de frequência dos idosos no programa de treinamento. Observou-se também a auto percepção dos participantes sobre a evolução dos indicadores avaliados durante os 6 meses do programa. Foi obtido um índice de satisfação de $80 \%$ em participar do projeto, melhora da condição de saúde, diminuição da pressão arterial, das dores e do uso de medicamentos, como aumento da capacidade funcional diária. Desta forma o programa se apresenta como uma ótima estratégia para o programa da saúde do idoso em diferentes regiões do Estado.

Palavras-chave: Envelhecimento, Programa de Saúde, Satisfação 


\begin{abstract}
The aim of study is postulate in the potentiality of a systematic activities program to elderly. Exploratory study with 83 elderly, both sex, between 60 and 85 years, introduced in a program called Active Life Program in Campos dos Goytacazes/RJ. Was used an interview made by researchers specifically to verify the perception of participants about health indicators, practice of activities, satisfaction, quality of life and pre-existence of chronic diseases 6 months after the start of participation in the program. The results

showed high index of frequency of elderly in the training program, other results of program potentiality were related to participants' selfperception about the evolution of evaluated indicators during the 6 months of program. $80 \%$ of satisfaction to participate of project, better health condition, decreases of blood pressure, the pain, and the use of medicines, as increase of daily functional capacity. In this way the program presents itself as an great strategy for the elderly health program in different regions of state.
\end{abstract}

Key words: Aging, Health Program, Satisfaction

\footnotetext{
${ }^{1}$ Especialista em Gerontologia e Qualidade de Vida FAVENI e MBA em Gestão de Projetos Institutos Superiores de Ensino do CENSA- ISECENSA - Rua Salvador Correa, 139, Centro, Campos dos Goytacazes, RJ, CEP: 28035-310, Brasil;

${ }^{2}$ Pesquisadora do Envelhecimento Ativo e Saudável - Member of International Fedaration on Ageing;

${ }^{3}$ Doutor em Ciências da Saúde - Faculdade São Judas Tadeu- FSTJ/RJ- Rua Clarimundo de Melo, 79 - Encantado, Rio de Janeiro, RJ, CEP: 20740-321, Brasil.

(*)e-mail: landim.hl08@gmail.com

Data de chegada: 01/06/2017 Aceito para publicação: 22/09/2017
}

Persp. online: hum. \& sociais aplicada., Campos dos Goytacazes, 20 (7), 82-89, 2017

seer.perspectivasonline.com.br 


\section{INTRODUÇÃO}

$\mathrm{Na}$ atualidade ter um olhar diferenciado e pratico sobre as questões do envelhecimento, é subsidiar os desafios profissionais e entidades, pois em um país como Brasil no qual a população de idosos aumenta e as perspectivas de envelhecimento não são tão evidentes para toda a população, que necessitam de cuidados e atenção redobrada, sempre com muitos desafios a serem vencidos. Os desafios a serem vencidos para Veras (2012) são: 1. Falta de políticas públicas que reúnam esforços no sentido de atendimento ao Idoso; 2 . As necessidades com programas destinados a preparar os cidadãos para envelhecer; 3. As dificuldades de se envelhecer com saúde; e 4. A população que envelhece cada vez mais sedentária, com hábitos de vida menos saudáveis.

O Envelhecimento populacional no Brasil vem crescendo há décadas e a cada década a projeção de crescimento da população com mais de 60 anos se eleva em maior número que as demais faixas etárias ou idades (VERAS 2001). Assis (2005) confirma essa realidade, ao descrever que:

"a longevidade da população é um fenômeno Mundial, e que o Brasil é um exemplo claro deste processo que se caracteriza pelo o aumento absoluto e relativo. A diminuição das taxas de fecundidade e mortalidade vem alterando a estrutura etária da população brasileira, gerando uma acentuada diminuição nas taxas de mortalidade, particularmente nos primeiros anos de vida".

O crescimento da população a partir dos 60 anos trouxe junto os desafios de envelhecer em um país que não criou e nem desenvolveu ao longo dos anos uma rede de proteção social para o envelhecimento saudável. Alves Júnior (2009 apud BRASIL 2012) afirma que "na segunda metade do século XX constata-se que a humanidade conquistou o desejado prolongamento da expectativa de vida através da evolução da medicina e áreas correlatas", porém contrapondo a celebração da causa, cria-se o desafio de repensar o desenvolvimento econômico e social, a fim de garantir a preservação da dignidade humana, afinal mesmo que o sistema não seja adequadamente preparado, todas as pessoas incluindo os idosos, precisam ter suas necessidades básicas satisfeitas e isso envolve questões como moradia, lazer, ocupação funcional e renda (SILVA e SANTOS, 2010).

O Programa de Envelhecimento Saudável - Vida Ativa, busca a melhoria da qualidade de vida em função da prática permanente e sistemática da atividade física voltada para as necessidades e a satisfação do Idoso, proporcionando uma melhora na capacidade funcional, pois segundo Alves Junior (2009) atividade física regular, auxilia no perfil social, cultura, na formação de grupos, além de uma interação motivadora, através da convivência de pessoas com o mesmo interesse e proposta de vida, como também dos processos de manutenção do tônus e da capacidade biológica e funcional deste indivíduo.

Desta forma o objetivo deste estudo é apresentar as potencialidades de um programa permanente de atividades físicas, denominado Programa Vida Ativa de Ginástica, para o envelhecimento saudável.

Persp. online: hum. \& sociais aplicada., Campos dos Goytacazes, 20 (7), 82-89, 2017

seer.perspectivasonline.com.br 


\section{METODOLOGIA}

Para responder ao estudo utilizaram-se procedimentos metodológicos que o caracterizam como sendo um estudo de caráter, exploratório. Participaram desta investigação 83 idosos, com idades acima dos 60 anos, de ambos os sexos, inseridos no Programa de Envelhecimento Saudável Vida Ativa desenvolvido no município de Campos dos Goytacazes/RJ. Foram incluídos no estudo todos os idosos matriculados no programa Vida Ativa que concordassem em participar voluntariamente através do termo de participação consentida e livre, e, foram excluídos os idosos que não pudessem participar integralmente do programa da Vida Ativa, ou que obtivessem mais de 3 faltas durante os 6 meses precedentes à coleta dados feita para a presente pesquisa.

Para obter as informações necessárias utilizou-se como instrumento uma entrevista elaborada pelos autores especificamente desenvolvida para atender aos objetivos propostos para esse estudo. Essa entrevista constava de 10 (dez) perguntas com indicadores de saúde, prática de atividades, satisfação, qualidade de vida e pré-existência de doenças crônicas. As entrevistas foram feitas individualmente 6 meses após a implantação do projeto, e, ocorriam antes do horário das atividades com duração aproximada de 10 minutos.

O programa Vida Ativa consiste de aulas sistemáticas três (3) vezes por semana com sessões de 60 minutos de exercícios físicos. Cada sessão de treinamento constava de 40 minutos de exercícios físicos contra resistidos, além de 20 minutos exercícios aeróbicos, como caminhadas em torno da praça onde se desenvolvia o projeto.

O desenvolvimento do tônus muscular era estimulado através de exercícios isométricos e isotônicos utilizando o peso do próprio corpo, exercícios de força muscular utilizando alteres de $2 \mathrm{~kg}$ e $1 \mathrm{~kg}$ realizando flexão e extensão do cotovelo, adução e abdução das pernas com pesos de $1 \mathrm{~kg}$ e 2 $\mathrm{kg}$, exercícios de equilíbrio estático e dinâmico através de trajeto a ser percorrido com marcações pelo chão em linhas retas e sinuosas, subidas e descidas através de obstáculos montados a uma altura máxima de $40 \mathrm{~cm}$ do chão e utilização do meio fio para caminhadas em busca do equilíbrio. Já a capacidade cardiorrespiratória era desenvolvida por meio de caminhadas com percursos de duração de 15 a 20 minutos totalizando cerca de 800 a $1000 \mathrm{~m}$. Também se buscava o desenvolvimento da coordenação motora fina e global dando-se ênfase a exercícios com utilização de bolas de borracha de vários diâmetros, flexibilidade e alongamento utilizando-se do sistema $3 \mathrm{~S}$ e elástico extensor.

Junto com as atividades físicas o programa ofertava palestras e bate papos informativos trimestralmente aos idosos, como por exemplo, os seminários "Desafios \& Diálogos para o Envelhecimento Saudável", que teve como temas: Envelhecimento Saudável começa pela Boca; Envelhecimento e Desafios da Sexualidade Ativa; Nutrição Funcional e seus benefícios para o Envelhecimento Saudável; O Idoso e seus Direitos assegurados em Lei, que despertavam grande interesse por parte dos alunos do programa sobre seus direitos assegurados em Lei e saúde preventiva. Todas as palestras e bate papos informativos ocorreram com especialistas da área de diferentes âmbitos profissionais, como, fisioterapeuta, nutricionista, psicólogo, advogado e médico, bem como encontros sociais com cafés da manhã, passeios culturais e caminhadas, que também eram promovidos mensalmente. Todas as atividades procuram melhorar a capacidade funcional dos idosos, seus sentimentos em relação sua autoestima, relacionamentos e a sociedade que estão inseridos.

Persp. online: hum. \& sociais aplicada., Campos dos Goytacazes, 20 (7), 82-89, 2017

seer.perspectivasonline.com.br 


\section{RESULTADOS}

Os resultados encontrados apresentam uma visão dos participantes sobre os primeiros 6 meses de participação no projeto Vida Ativa, tendo como primeiro resultado a observação do nível de engajamento de seus participantes, uma vez que, dos 83 idosos inscritos no programa apenas cinco não realizaram a entrevista por terem mais de 3 faltas durante a investigação. Tal fato por si só demonstra o quanto a participação lhes trazia satisfação, além dos benefícios descritos pela literatura e utilizados como respaldo para esta investigação.

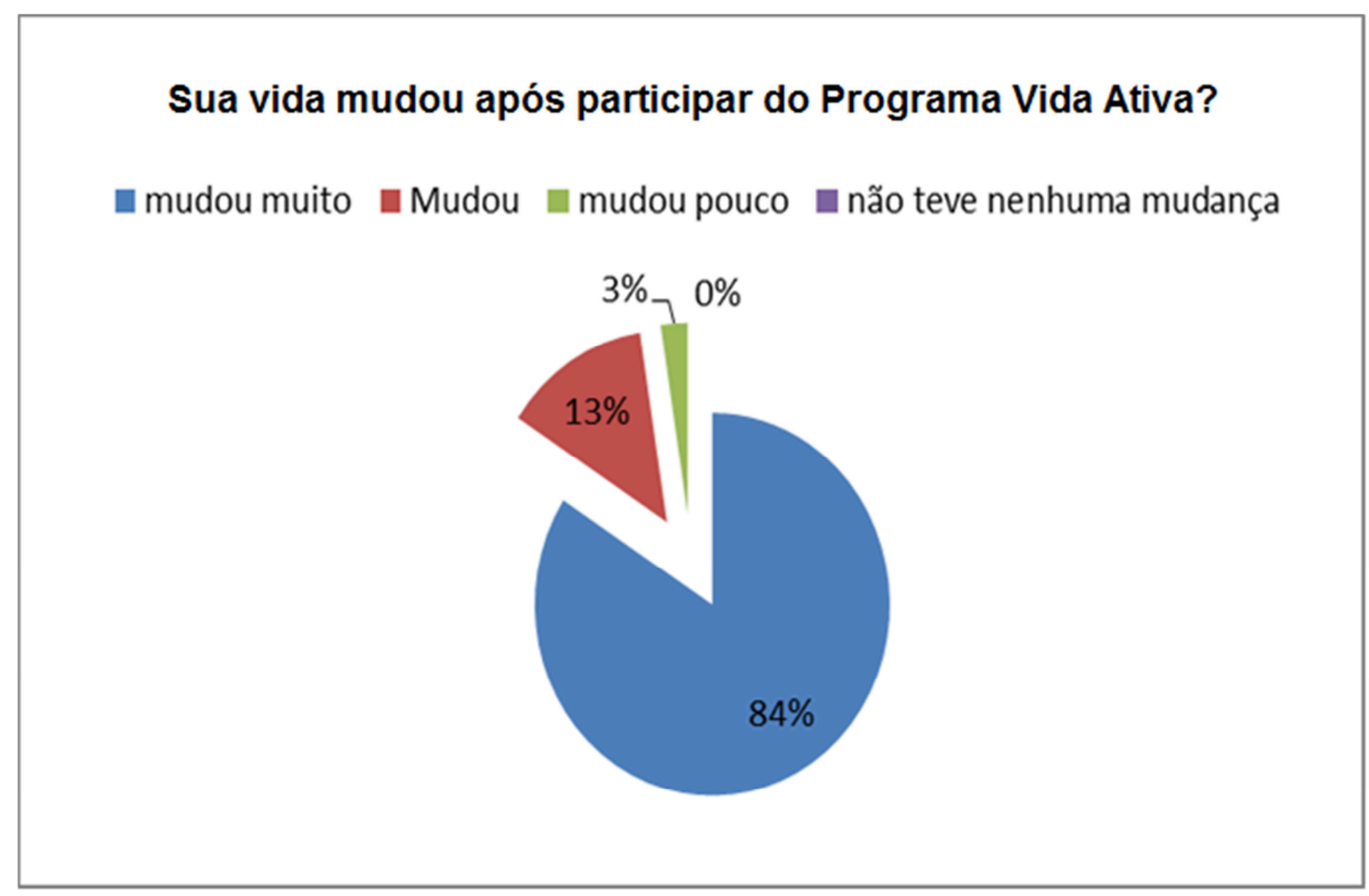

Figura 1: Apresentação do percentual de respostas dadas sobre a percepção do impacto da participação no programa Vida Ativa.

Para os entrevistados, no total de 83 idosos, todos participantes do Programa de Envelhecimento Saudável Vida Ativa - 97\% declararam que ocorrem mudança do estilo de vida, elevando sua participação no dia a dia, e 3\% declararam que foi uma mudança pequena. Os dados corroboram com diversos autores que afirmam ser a atividade física um dos elementos de grande importância para o envelhecimento saudável. Toscana e Oliveira (2009 apud FURTADO, 2012) afirmam que "O exercício é a grande alternativa para a longevidade e que o estilo de vida saudável está associado a prática de atividades físicas", corroborando, Furtado (2012) cita que "Ao praticar exercícios, os idosos podem conservar e recuperar sua capacidade funcional repercutindo positivamente em sua saúde".

Persp. online: hum. \& sociais aplicada., Campos dos Goytacazes, 20 (7), 82-89, 2017 


\section{Você passou a ter mais disposição para as suas atividades diárias após estar inserida no programa de atividades?}

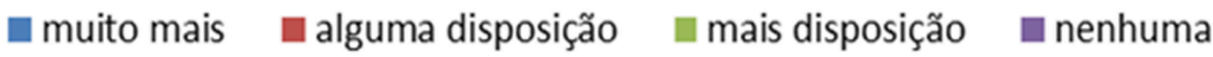

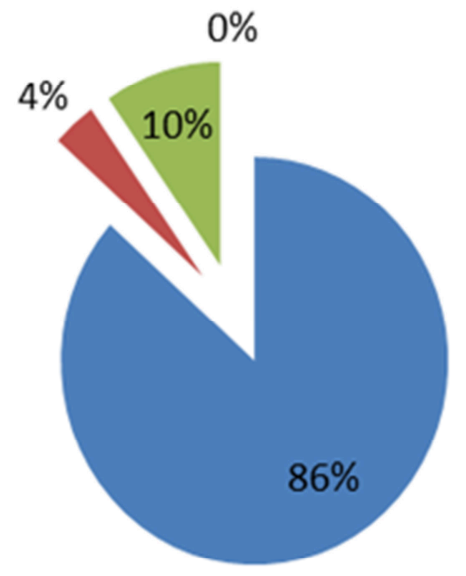

Figura 2: Percentil de respostas dadas sobre as possíveis mudanças na disposição para as suas atividades diárias após o ingresso no programa.

Confirmando os benefícios da atividade física atrelada ao aumento da capacidade funcional dos Idosos ativos, participantes do Programa de Envelhecimento Saudável Vida Ativa, 86\% confirma que elevou sua disposição para as suas atividades diárias, obtendo disposição para ir ao mercado, subir escada e andar pela casa, saindo do sedentarismo e se tornando cada vez mais ativo e independente em suas ações. Sendo ainda $10 \%$ que responderam ter mais disposição e $4 \%$ com alguma disposição.

Segundo Okuma (2002 apud FURTADO, 2012) “O exercício é reconhecido como forte aliado no processo de envelhecimento, suavizando as alterações como: diminuição de força muscular, do equilíbrio, a flexibilidade e da resistência aeróbica". 


\section{Dentre as doenças pré-existentes qual ou quais você possuí?}

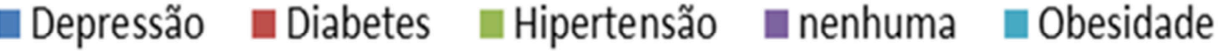

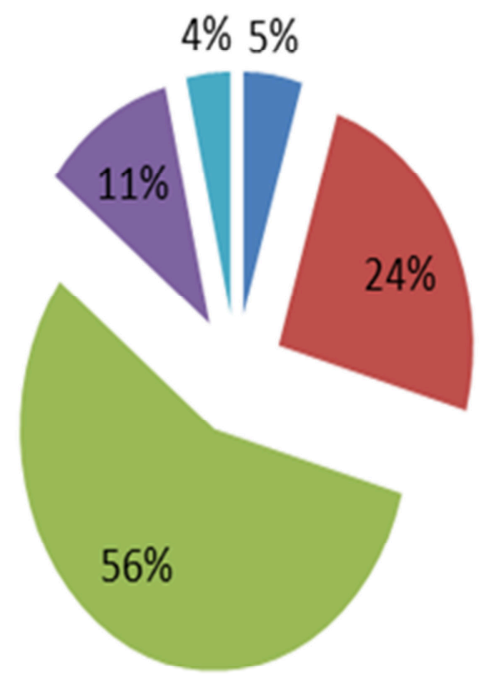

Figura 3: Apresentação do percentual de doenças pré-existentes relatadas pelos idosos participantes do estudo.

O sedentarismo é um facilitador para o aparecimento da Diabetes Mellitus, patologia que vem acompanhada de pouca irrigação periférica, amputações de membros inferiores, estado de incapacidade funcional e altas doses de medicação para obtenção de seu controle, como a Obesidade que desencadeia as duas anteriores redundando em uma síndrome metabólica além da depressão, fruto muitas vezes da perda de capacidade funcional, falta de ocupação do tempo e falta da integração social (BUSKAMAN, 2012).

No grupo investigado observou se que $56 \%$ de idosos tem hipertensão, $24 \%$ com Diabetes, $5 \%$ com quadro de depressão e $4 \%$ de obesos, Como também tinham 11\% dos participantes que não possuíam nenhuma patologia pregressa. 


\title{
Houve melhora comprovada do seu quadro clínico?
}

\author{
grande melhora $\square$ alguma $\quad$ pouca $\quad$ nenhuma
}

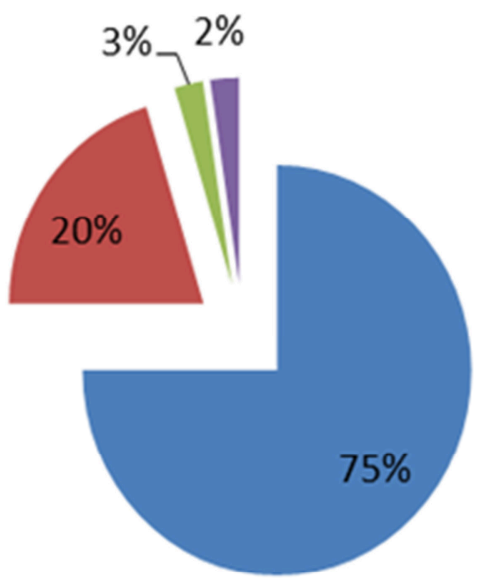

Figura 4: Percentis relativos à percepção dos idosos sobre a evolução do seu quadro clínico a partir do ingresso no programa Vida Ativa.

A percepção da melhora do quadro clínico é observada por $75 \%$ dos participantes, isto é, 63 dos idosos participantes, se sente menos impactados de uma forma ou outra com relação a sua doença, portanto mais da metade, apontando um dado muito positivo e postulando a importância da influência das atividades físicas inerentes ao Programa Vida Ativa como "grande melhora", já 20\% representando 17 idosos afirmam que obtiveram melhora em seu quadro clínico. Esse quadro de melhora em torno de $95 \%$ dos participantes ocorre pela prática da atividade física sistemática, isto é, mesmo modelo de estimulo semanalmente, ao qual oportuniza eficiências funcionais e biológicas, mas também mudança no estilo de vida, principalmente pela proposta do programa, que desenvolve a mente com informações que geram e capacitam pessoas no seu dia a dia. 


\section{Caso sua resposta na pergunta anterior tenha sido "sim", marque a opção que se assemelha a seu caso de doença.}

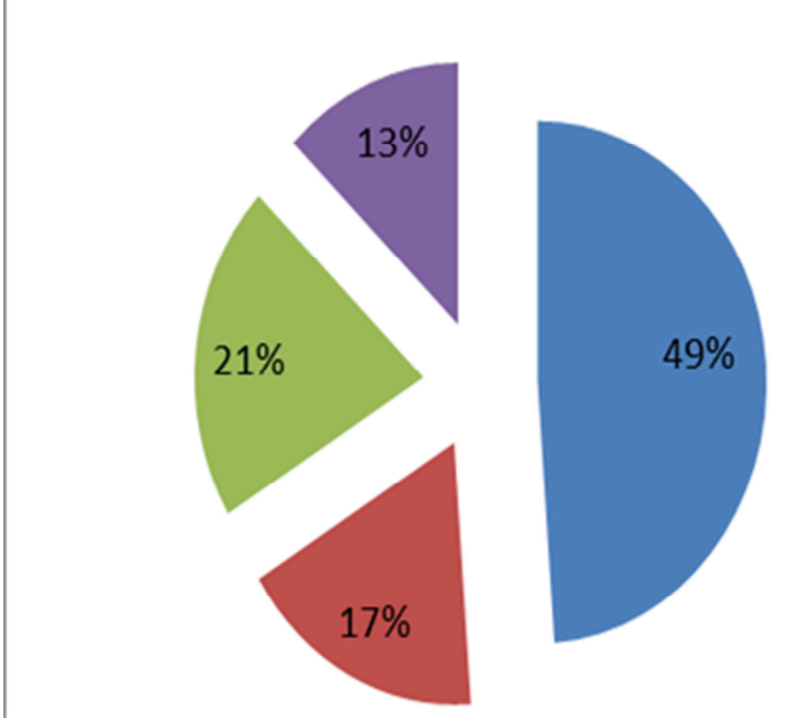

Diminuição da PA

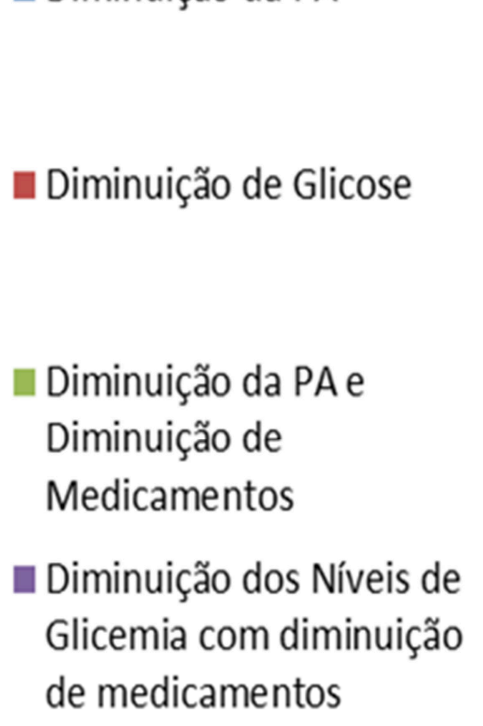

Figura 5: Respostas dadas sobre a especificidade da melhora relatada pelos idosos a a partir do ingresso no programa Vida Ativa.

Apesar de não ter sido solicitado aos idosos exames clínicos que comprovassem as informações dadas no instrumento do estudo, a mesma torna-se confiável uma vez que todos os participantes do estudo possuíam autonomia funcional preservada, sendo os mesmos responsáveis pelo controle de suas medicações. Assim, torna-se relevante a informação referente à diminuição de medicamentos aos quais eram submetidos em tratamentos clínicos para controle da doença préexistente como por exemplo, é o caso dos indivíduos com hipertensão arterial, em que $49 \%$ dos entrevistados confirmam a diminuição dos níveis pressóricos, se mantendo mais controlada, 13\% dos entrevistados informam a diminuição dos índices de glicemia e também da diminuição dos medicamentos que utilizavam para manter estes níveis, com relação ao percentual de hipertensos que diminui sua pressão arterial e também o usos de medicamentos, chega a $21 \%$ ou seja 18 idosos apontam nesse sentido um quadro de melhoria.

\section{CONCLUSÃO}

A atividade física é comprovadamente um dos aliados mais fortes do envelhecimento saudável. Os resultados encontrados na presente pesquisa mostram que um programa permanente e sistemático de atividades físicas, associado ao aumento da interação social e conscientização sobre direitos e deveres dos idosos promoveram após 6 meses mudanças positivas em diferentes aspectos das vidas dos idosos participantes do estudo.

Esses resultados também permitem concluir que programas como o descrito nesse estudo podem ser implantados como uma política pública de promoção do envelhecimento ativo e saudável, uma vez que os resultados indicam que além de promover mudanças positivas no estilo de

Persp. online: hum. \& sociais aplicada., Campos dos Goytacazes, 20 (7), 82-89, 2017 
vida e disposição dos idosos, também impactam em aspectos clínicos pré-existentes, resultando em redução no uso de medicamentos e de outros aspectos relacionados à saúde dessa crescente parcela da população.

Os primeiros estudos e ações no Brasil, junto aos idosos, data da década de 60, através do SESC (Serviço Social do Comércio). Há, portanto, um grande hiato entre ações e investimentos públicos com foco no envelhecimento saudável, seja na rede primária de atendimento, seja no planejamento e execução de políticas públicas que incluam a atividade física como um dos fatores preponderantes para o Envelhecimento Saudável. Desta forma seria importante relacionar os projetos existentes com os benefícios encontrados e assim realizar uma proposta governamental coerente e coesa.

\section{REFERÊNCIAS}

JUNIOR, Edmundo. Envelhecimento e vida saudável. apicuri, 2009.

ASSIS, Mônica. Envelhecimento ativo e promoção da saúde: reflexão para as ações educativas com idosos. Revista APS, v.8, n.1, p.15-24, jan./jun. 2005.

BUSKMAN, Rodrigo. Atrasando o Relógio Biológico. In FRANCISCO, Cristiane Brasil (org). Viver é a melhor opção... Envelhecer faz parte, Capitulo 6, Rio de Janeiro, QUARTET, 2012.

CHAIMOWISCZ e CAMARGOS, A saúde do Idoso no Brasil. In FRANCISCO, Cristiane Brasil (org). Viver é a melhor opção... Envelhecer faz parte, Capitulo 2, Rio de Janeiro, QUARTET,2012.

FURTADO, Hélio. Atividade Física e Envelhecimento. In FRANCISCO, Cristiane Brasil (org). Viver é a melhor opção... Envelhecer faz parte, Capitulo 8,. Rio de Janeiro, QUARTET, 2012.

IBGE- Instituto Brasileiro de Geografia e Estatística. Censo Demográfico 2010, Indicadores Sociais Municipais -População residente total de 60 anos de Idade ou maishttp:// www.cidades.ibge.gov.br/xtras/temas.php?lang $=\& \operatorname{codmun}=330100 \&$ idtema $=79 \&$ search $=$ riodejaneiro|campos-dos-goytacazes|censo-demografico-2010:-resultados-do-universo-indicadoressociaismunicipais--disponível em 17 de outubro de 2015, as 16h.

JAMILI, Jorge. Tratamentos alternativos e medicina antienvelhecimento. In FRANCISCO, Cristiane Brasil (org). Viver é a melhor opção... Envelhecer faz parte, Capitulo 7. Rio de Janeiro, QUARTET, 2012.

Silva, Aila Cristina dos Santos; Santos Iraci dos. Promoção do autocuidado de idosos para o envelhecer saudável: aplicação da teoria de Nola Pender. Enferm. Florianópolis, 2010 Out-Dez; v.19, n.4, pp. 745-53.

VERAS, Renato. Velhice Numa perspectiva de futuro saudável, Rio de Janeiro - UERJ, UnATI, 2001.

VERAS, Renato. Idoso: A aprendizagem continuada e a função social das UnATIS. In FRANCISCO, Cristiane Brasil (org). Viver é a melhor opção... Envelhecer faz parte, Capitulo 13, Rio de Janeiro, QUARTET, 2012.

Persp. online: hum. \& sociais aplicada., Campos dos Goytacazes, 20 (7), 82-89, 2017 seer.perspectivasonline.com.br 